\title{
PROPRIEDADES FÍSICAS E MECÂNICAS DA MADEIRA DE Toona ciliata EM DIFERENTES IDADES
}

\author{
Rafael Leite Braz ${ }^{1}$, José Tarcísio da Silva Oliveira², Brunela Pollastrelli Rodrigues ${ }^{3}$, \\ Marina Donária Chaves Arantes ${ }^{2}$ \\ ${ }^{1}$ Eng. Florestal, M.Sc., Doutorando em Engenharia Florestal, UFPR, Curitiba, PR, Brasil - rafaellbraz@ yahoo.com.br \\ ${ }^{2}$ Eng. Florestal, Dr., Depto. Ciências Florestais e da Madeira, UFES, Jerônimo Monteiro, ES, Brasil - jtsilva @npd.ufes.br; \\ mdonariac@hotmail.com \\ ${ }^{3}$ Eng $^{\text {a }}$. Florestal, M.Sc., Doutoranda em Ciências Florestais, UFES, Jerônimo Monteiro, ES, Brasil - brunelapollastrelli@ yahoo.com.br
}

Recebido para publicação: 22/01/2013 - Aceito para publicação: 10/09/2013

\begin{abstract}
Resumo
O presente trabalho teve como objetivo caracterizar a madeira de árvores de Toona ciliata (cedro australiano) aos 6,12 e 18 anos de idade, provenientes de um plantio florestal na região de Venda Nova do Imigrante, ES. Foram avaliadas as propriedades físicas quanto à densidade básica e à retratibilidade e as propriedades mecânicas quanto à resistência à compressão axial e ao módulo de ruptura e módulo de elasticidade à flexão estática da madeira. Foram utilizadas três árvores para cada idade, das quais foram retiradas toras, que foram desdobradas em pranchas radiais para retirada de corpos de prova, ensaiados de acordo com as normas MB 26/40 (ABNT, 1940) e NBR 7190 (1997). Observou-se o aumento da densidade básica e a redução das contrações tangencial e volumétrica e do fator anisotrópico em função do aumento da idade. O uso da madeira deve ser limitado em situações que exijam elevada resistência mecânica e na fabricação de produtos com elevada estabilidade dimensional. Deve-se optar por árvores com idades superiores, viabilizando a aplicabilidade da madeira com qualidade para diversos fins.

Palavras-chave: Cedro australiano; resistência da madeira; estabilidade dimensional.
\end{abstract}

\begin{abstract}
Physical and mechanical properties of wood of Toona ciliata at different ages. The objective of the present study was to characterize the wood of trees Toona ciliata at 6,12 and 18 years old, coming from forest plantations in the region of Venda Nova do Imigrante at Espírito Santo State in Brazil. We evaluated the physical properties as the specific gravity and shrinkage, and the mechanical parameters in relation to the compressive strength and modulus of rupture and elasticity in static bending. We use tree logs that were sawed and prepared the samples for analysis according to standards MB 26/40 (ABNT, 1940) e NBR 7190 (1997). We observed an increase in specific gravity and decrease of tangential and volumetric shrinkages and the anisotropic factor with the increase for the age. The use of this wood should be limited in situations which high mechanical strength is required and manufacture of products with high dimensional stability. We should opt for more aged trees, allowing the applicability of quality wood for various purposes.

Keywords: Australian cedar; wood resistance; dimensional stability.
\end{abstract}

\section{INTRODUÇÃO}

A madeira é um material biológico, heterogêneo e bastante complexo, com elevada variabilidade em relação às suas características e propriedades. O estudo de suas propriedades físicas e mecânicas se faz necessário para aumentar o conhecimento a respeito do seu potencial de utilização, adequando seu uso, e ainda como alternativa para substituição de outras madeiras. Diferenças entre as propriedades da madeira estão presentes tanto entre espécies como entre indivíduos de mesma espécie e até entre diferentes regiões de um mesmo indivíduo (BURGER; RICHTER, 1991).

Segundo Shimoyama (1990), a densidade está relacionada a muitas propriedades e características tecnológicas fundamentais para a produção e utilização dos produtos florestais, sendo um dos parâmetros mais importantes entre as propriedades físicas da madeira, pois apresenta relação com as demais propriedades dela. Normalmente, quanto maior a densidade da madeira, maiores serão as propriedades 
mecânicas. Segundo Sturion et al. (1987), madeiras com alta densidade são adequadas para construção civil e para produção de energia. Gonzaga (2006) acrescenta que madeiras densas são adequadas para uso no solo, como estacas, mourões, dormentes e construção de pontes.

Outra importante propriedade da madeira é a retratibilidade, que, segundo Mori et al. (2003), varia muito de uma espécie para outra e com o modo de secagem, podendo inchar ou contrair de acordo com a umidade relativa do meio em que se encontra. Os mesmo autores ainda ressaltam que, quanto menores as contrações, melhores serão as suas propriedades. Essa propriedade deve ser considerada quando se recomenda madeira para usos que exijam boa estabilidade dimensional, evitando o aparecimento de fendas e empenos.

As propriedades mecânicas definem o comportamento da madeira quando submetida a esforços de natureza mecânica, permitindo compará-la com outras madeiras de propriedades conhecidas e por analogia indicar as provas adicionais necessárias para conhecer sua utilização (STANGERLIN et al., 2008). Os valores das propriedades mecânicas variam de acordo com a espécie, umidade da madeira, densidade e tempo de duração da carga durante o ensaio mecânico, entre outros fatores. O módulo de ruptura (MOR) e o módulo de elasticidade (MOE) são dois parâmetros normalmente avaliados em testes de flexão estática, sendo o módulo de elasticidade de maior importância na caracterização tecnológica da madeira, representando a resistência do material quando submetido a uma carga aplicada (SCANAVACA JÚNIOR; GARCIA, 2004).

De acordo com Raymond (2000), as propriedades de resistência e estabilidade dimensional são importantes na qualificação da madeira para fabricação de móveis, pois elas são realçadas nos componentes estruturais, destacando-se a importância do MOE e do MOR, observando-se valores mais elevados em madeiras de lenho adulto e de maior idade.

Uma das espécies promissoras para a obtenção de produto de madeira sólida de qualidade como fonte para o abastecimento das indústrias no setor florestal é o cedro australiano, Toona ciliata M. Roem. Var. australis (F. Muell.) Bahadur, pertencente à família Meliaceae, oriunda das regiões tropicais da Austrália. A espécie apresenta elevado potencial para silvicultura comercial (ARES; FOWNES, 2000), excelente substituta dos cedros nativos ao fornecimento de madeira avermelhada, servindo como alternativa em reflorestamento.

Segundo Pinheiro et al. (1994), a árvore pode alcançar, aos três anos idade, uma altura de 8,0 m e 15,0 cm de diâmetro, além de não apresentar problemas com ataques de pragas, principalmente pela Hypsyphila robusta, que não tem ocorrência natural na América (CUNNINGHAM et al., 2005). Em relação à durabilidade natural da madeira, Gonçalves e Oliveira (2006) constataram para a madeira de Toona ciliata um desgaste médio, após 45 dias de exposição a cupins de madeira seca. Almeida (2010) classificou a madeira como moderadamente resistente.

Por ser uma espécie madeireira com propriedades tecnológicas interessantes, a espécie Toona ciliata é vista como uma árvore de grande potencial, podendo resultar em usos madeiráveis nobres. Para Ziech (2008), a madeira de cedro australiano apresenta características tecnológicas semelhantes à de espécies nobres brasileiras, podendo se tornar, no futuro, uma alternativa ao uso destas em determinadas utilizações. De acordo com o mesmo autor, ela apresenta madeira com textura média, linhas vasculares retilíneas, cheiro agradável ao corte, sem gosto, superfície radial pouco lustrosa, madeira macia ao corte e cerne bege-rosado, distinto do alburno marrom-claro.

A madeira pode ser empregada na produção de laminados, móveis, produção de caixas de charutos, instrumentos musicais e outras finalidades especiais (LAMPRECHT, 1990). De acordo com Soragi (2009), madeira de Toona ciliata M. Roem. apresenta superfícies usinadas de boa qualidade, mostrando-se apta para confecção de produtos sólidos. Sá (2009), ao estudar a madeira de Toona ciliata na manufatura de painéis compensados OSB, aglomerados e cimento-madeira, identificou um grande potencial dessa madeira, ressaltando o cimento-madeira, que atingiu todos os valores satisfatórios, indicando a viabilidade técnica de seu uso como matéria-prima na confecção de painéis cimento-madeira de boa qualidade, independentemente da localidade e da idade. Trianoski (2010) concluiu que a espécie Toona ciliata possui potencialidade para a produção de painéis de madeira aglomerada.

Gonçalez et al. (2006) afirmaram que a qualidade da madeira se refere à sua capacidade de atender aos requisitos necessários para fabricação do produto, ou, ainda, à combinação das características físicas, mecânicas, químicas e anatômicas de uma árvore, que permite a melhor utilização da madeira. 
A madeira de Toona ciliata M. Roemer pode ser considerada mais uma opção de uso, com grande potencial madeireiro. Visto que existem poucos estudos sobre a espécie, a qualificação das propriedades da madeira torna-se necessária para sua adequação aos mais diversos usos. Nesse contexto, o presente trabalho tem como objetivo determinar algumas propriedades físicas e mecânicas da madeira de Toona ciliata $\mathrm{M}$. Roemer aos 6,12 e 18 anos de idade, para um melhor aproveitamento quanto ao seu uso.

\section{MATERIAL E MÉTODOS}

A madeira utilizada no presente estudo foi proveniente de árvores de Toona ciliata M. Roemer (cedro australiano), com idades de 6, 12 e 18 anos, procedentes de plantios florestais de Venda Nova do Imigrante, região serrana do estado do Espírito Santo. A região apresenta um relevo montanhoso e ondulado, com temperatura média que varia de 12 a $24{ }^{\circ} \mathrm{C}$, com umidade relativa do ar em torno de $85 \%$ (média) e índice pluviométrico de aproximadamente $1534 \mathrm{~mm} / \mathrm{ano}$. O clima é tropical, com duas estações bem definidas (seca e úmida) e vegetação predominante de Mata Atlântica.

Foram utilizadas três árvores para cada idade, retiradas aleatoriamente do povoamento. De cada árvore selecionada foi retirada uma tora da base de $3 \mathrm{~m}$ de comprimento, que foram encaminhadas para o Laboratório de Ciência da Madeira (LCM), Departamento de Ciências Florestais e da Madeira, da Universidade Federal do Espírito Santo (UFES), localizado no município de Jerônimo Monteiro, ES.

Para determinação da densidade básica da madeira, utilizou-se a MB 26/40 da Associação Brasileira de Normas Técnicas (ABNT) (1940), confeccionando-se corpos de prova nas dimensões de $2,0 \times 2,0 \times 3,0 \mathrm{~cm}$, sendo a maior medida orientada no sentido longitudinal. As amostras foram saturadas em um dissecador com água e foi realizada a aplicação de vácuo intermitente. Em seguida calculou-se o volume imerso em água para cada corpo de prova, pelo método da balança hidrostática. Após a secagem natural, as amostras foram colocadas em uma estufa de ventilação forçada, a uma temperatura de $103{ }^{\circ} \mathrm{C}$ $\pm 2{ }^{\circ} \mathrm{C}$, até atingirem massa constante, determinado assim a sua massa seca. A densidade básica da madeira foi obtida pela razão entre a massa seca e o volume saturado.

A retratibilidade foi calculada pela diferença das dimensões dos mesmos corpos de prova utilizados para a densidade básica. As medidas foram tomadas quando estavam na condição completamente saturados para secos, realizada de acordo com a norma NBR 7190 (ABNT, 1997). Determinaram-se também as contrações lineares, volumétricas e o coeficiente de anisotropia.

Para a determinação das propriedades mecânicas, as dimensões dos corpos de prova seguiram a norma ABNT MB 26/40 (1940), com ensaios realizados segundo a norma NBR 7190 (ABNT, 1997). Foram confeccionados corpos de prova com dimensões de $2,0 \times 2,0 \times 30,0 \mathrm{~cm}$, para a realização dos ensaios de flexão estática da madeira, a fim de se obter o módulo de elasticidade (MOE) e o módulo de ruptura (MOR), e os corpos de prova com dimensões de 2,0 x 2,0 x 3,0 cm para o ensaio de resistência à compressão axial. Os ensaios mecânicos foram realizados em uma máquina universal de ensaios com sistema de aquisição de dados automatizado, com a madeira a $12 \%$ de umidade.

Os valores referentes às propriedades física e mecânica da madeira de Toona ciliata foram tabulados e, para se avaliar o grau de significância das variáveis analisadas e a variação existente entre as idades, realizou-se uma análise de variância e, quando significativo, aplicou-se o teste Tukey a 5\% de probabilidade para a comparação de médias.

\section{RESULTADOS E DISCUSSÃO}

\section{Propriedades físicas}

Os valores médios da densidade básica da madeira e as contrações tangencial, radial e volumétrica para a madeira de Toona ciliata, aos 6,12 e 18 anos de idade, são demonstrados na tabela 1.

$\mathrm{O}$ valor médio para a densidade básica foi de $0,318 \mathrm{~g} / \mathrm{cm}^{3}$. Estatisticamente, as árvores com idades de 12 e 18 anos não apresentaram diferenças significativas, apenas árvores com 6 anos de idade diferenciaram-se das demais. Observou-se uma tendência do crescimento dos valores para densidade básica em função do aumento da idade. Mendes (1993) e Calegari et al. (2004) ressaltaram que a maior densidade da madeira em idades mais avançadas pode ser justificada pela maior proporção de madeira adulta, em relação à madeira juvenil. Panshin e Zeeuw (1980) descrevem que a densidade básica em 
função da idade da árvore em geral aumenta rapidamente durante o período juvenil, depois mais lentamente, até atingir a maturidade, quando permanece mais ou menos constante.

Tabela 1. Valores médios da densidade básica e os coeficientes de retratibilidade da madeira de Toona ciliata tomados aos 6, 12 e 18 anos de idade.

Table 1. Average values of the specific gravity and the shrinkage coefficient of Toona ciliata wood at 6, 12 and 18 years old age.

\begin{tabular}{lcccc}
\hline \multirow{2}{*}{ Características } & \multicolumn{3}{c}{ Idade (anos) } & Média geral \\
\cline { 2 - 4 } & $\mathbf{6}$ & $\mathbf{1 2}$ & $\mathbf{1 8}$ & \\
\hline Densidade básica $\left(\mathrm{g} / \mathrm{cm}^{3}\right)$ & $0,293 \mathrm{~b}^{*}$ & $0,322 \mathrm{a}$ & $0,338 \mathrm{a}$ & 0,318 \\
& $(0,021)(7,111)^{* *}$ & $(0,018)(5,518)$ & $(0,026)(7,606)$ & $(0,028)(8,918)$ \\
Contração tangencial $(\%)$ & $11,18 \mathrm{a}$ & $8,75 \mathrm{~b}$ & $7,84 \mathrm{c}$ & 9,19 \\
& $(1,25)(11,32)$ & $(1,08)(12,38)$ & $(0,86)(11,01)$ & $(1,75)(19,10)$ \\
Contração radial $(\%)$ & $3,32 \mathrm{~b}$ & $4,33 \mathrm{a}$ & $3,31 \mathrm{~b}$ & 3,65 \\
& $(0,61)(18,32)$ & $(0,72)(16,57)$ & $(0,66)(19,90)$ & $(0,81)(22,24)$ \\
Contração volumétrica $(\%)$ & $15,67 \mathrm{a}$ & $14,73 \mathrm{a}$ & $13,09 \mathrm{~b}$ & 14,45 \\
Coeficiente de retratibilidade & $(2,04)(13,25)$ & $(1,29)(8,75)$ & $(1,50)(11,46)$ & $(1,94)(13,94)$ \\
volumétrica & $0,56 \mathrm{a}$ & $0,53 \mathrm{a}$ & $0,47 \mathrm{~b}$ & 0,52 \\
Fator anisotrópico $(\mathrm{T} / \mathrm{R})$ & $(0,07)(13,35)$ & $(0,05)(8,75)$ & $(0,05)(11,46)$ & $(0,07)(13,44)$ \\
& $3,50 \mathrm{a}$ & $2,09 \mathrm{~b}$ & $2,49 \mathrm{~b}$ & 2,67 \\
& $(0,80)(22,82)$ & $(0,50)(24,15)$ & $(0,70)(28,01)$ & $(0,89)(33,16)$ \\
\hline
\end{tabular}

*: As médias seguidas pela mesma letra não diferem estatisticamente entre si (Tukey, p >0,05). **: Valores entre parênteses são o desvio padrão e o coeficiente de variação, respectivamente.

Conforme a classificação da densidade básica da madeira proposta pelo Forest Products Laboratory (1973), pode-se afirmar que os valores encontrados para a madeira das árvores de cedro australiano selecionadas para o estudo são classificados como madeira leve. Para Hellmeister (1982), a densidade é a propriedade física mais significativa para caracterizar a madeira para a utilização em construções civis, fabricação de chapas e móveis. Diversos autores afirmaram que a densidade é ponto de partida no estudo da madeira para diversas utilizações, uma vez que todas as demais propriedades estão relacionadas com ela (SHIMOYAMA; BARRICHELO, 1991; HUMPHREYS; CHIMELO, 1992).

Os valores encontrados para a densidade básica da madeira estão próximos aos de outras pesquisas, como de Ziech (2008), que, ao estudar a madeira de cedro australiano com aproximadamente 4 anos de idade, encontrou um valor médio geral para a densidade básica de $0,306 \mathrm{~g} / \mathrm{cm}^{3}$. Pereyra et al. (2006), ao estudarem a madeira de cedro australiano oriunda de plantações comerciais na Argentina, entre 18 e 20 anos, obtiveram um valor de $0,336 \mathrm{~g} / \mathrm{cm}^{3}$ para a densidade básica. Já Nassur (2010) encontrou um valor médio de $0,314 \mathrm{~g} / \mathrm{cm}^{3}$ para essa propriedade da madeira de cedro australiano com 18 anos de idade.

Para as contrações radial, tangencial e volumétrica, os valores médios encontrados foram 3,65\%, $9,19 \%$ e $14,45 \%$, respectivamente. Uma menor diferença entre as contrações radiais e tangenciais indicam uma madeira mais estável. Estatisticamente, os valores para a contração tangencial diferenciaram entre si em função da idade, com decréscimo com o aumento da idade. Observou-se, na direção radial, que a contração na madeira aos 12 anos foi significativamente mais elevada do que aos 6 e 18 anos. Em relação à contração volumétrica, os valores das contrações foram significativamente mais altos para a madeira aos 6 e 12 anos, com redução das contrações em função do aumento da idade. O coeficiente de retratibilidade volumétrica foi estatisticamente superior para as madeiras aos 6 e 12 anos, decrescendo com o aumento da idade.

A variação da contração radial, tangencial e volumétrica, bem como o fator anisotrópico para a madeira em estudo pode ser visualizada em função das idades por meio da figura 1.

Os valores encontrados estão coerentes com os citados na literatura, em que Ziech (2008), ao estudar a madeira de cedro australiano com aproximadamente quatro anos de idade, encontrou os valores de 2,8, 8,01 e 15,52\% para as contrações radial, tangencial e volumétrica, respectivamente. Nassur (2010) encontrou valores médios de 3,22\% para contração radial, 7,78\% para contração tangencial e 11,00\% para volumétrica. Kollmann e Côté Júnior (1968) ressaltam que as contrações tangenciais e radiais da madeira desde o estado verde até seco em estufa devem estar nos intervalo de 3,5\% a $15,0 \%$ e $2,4 \%$ a $11,0 \%$, respectivamente. 


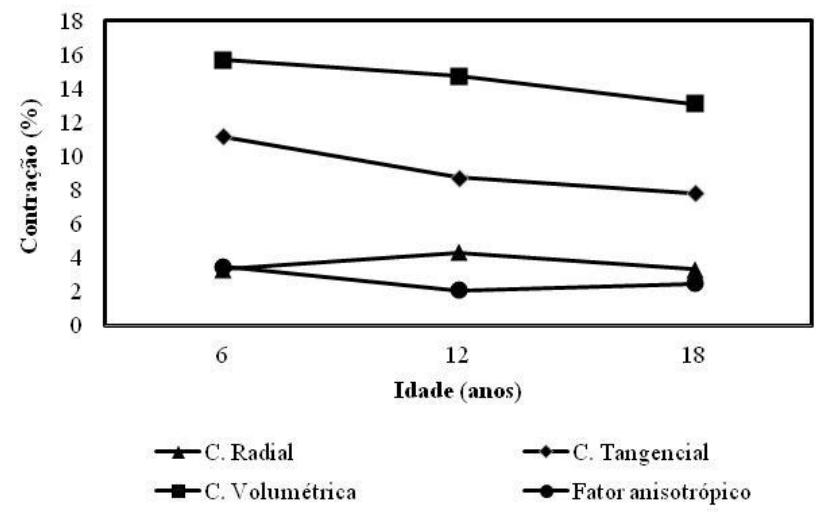

Figura 1. Variação das contrações radiais, tangenciais e volumétricas e fator anisotrópico em função da idade para a madeira de Toona ciliata.

Figure 1. Variation of radial, tangential and volumetric shrinkages and anisotropic factor according to age to the wood of Toona ciliata.

A alteração dimensional na direção tangencial foi superior àquela da direção radial, como já era o esperado. Panshin e Zeeuw (1980) afirmaram que a maior alteração dimensional se manifesta na direção tangencial aos anéis de crescimento, seguida pela dimensão radial e, praticamente, desprezível na direção longitudinal. Elevadas contrações da madeira são características indesejáveis dependendo da sua utilização. Oliveira (1997) mencionou que a retratibilidade volumétrica, apesar de expressar a variação total, ocorrida na variação higroscópica, são as contrações lineares que ocorrem ao longo dos planos de orientação da madeira na maioria das vezes mais importantes, porque, pelo fato de serem diferentes, tornam a madeira um material anisotrópico.

O fator anisotrópico é a relação direta entre a retratibilidade na direção tangencial e radial. Segundo Oliveira et al. (2010), a situação ideal, raramente encontrada, seria aquela na qual as tensões decorrentes da natureza anisotrópica se anulariam segundo as direções em que a retratibilidade se manifestasse. O valor médio encontrado no presente trabalho foi de 2,67. A madeira aos seis anos de idade apresentou o maior fator anisotrópico. Verificou-se que esse parâmetro apresentou uma tendência decrescente com o aumento da idade, consequentemente, baixos valores para o fator anisotrópico indicam uma elevada estabilidade dimensional da madeira, o que tende a reduzir defeitos como os empenamentos e fendilhamentos.

O valor encontrado no presente trabalho para o fator anisotrópico está de acordo com os valores encontrados por Nassur (2010), que, ao estudar madeira de cedro australiano, encontrou um fator anisotrópico de 2,71. Já Ziech (2008) encontrou valores médios variando de 2,63 a 4,91 na direção medula-casca. Durlo e Marchiori (1992) apresentaram o seguinte critério de classificação da madeira quanto ao fator anisotrópico: 1,2-1,5 - considerado excelente, ocorrendo em madeira de cedro, sucupira, mogno e balsa, entre outras; 1,5-2,0 - normal, exemplificados por ipê, pinus, araucária, peroba-rosa e teca, entre outras espécies; e acima de 2,0 - ruim, que pode ocorrer em madeiras de araucária, imbuia, álamo e jatobá, entre outras espécies.

Ao comparar o valor encontrado com as classes mencionadas pelos autores anteriormente citados, a madeira de Toona ciliata em geral se classifica como ruim, o que indica uma baixa estabilidade dimensional e que devem ser tomadas algumas precauções quanto ao uso final da madeira. Entretanto, observa-se que a idade da madeira influenciará na obtenção do produto final desejado, uma vez que, aos 12 anos, por exemplo, o fator anisotrópico ficou próximo de 2 , indicando uma qualidade superior da madeira nessa faixa de idade.

\section{Propriedades mecânicas}

A tabela 2 demonstra os valores médios para os ensaios mecânicos de compressão axial, o MOE e o MOR à flexão estática da madeira de Toona ciliata a 12\% de umidade aos 6, 12 e 18 anos de idade. 
Tabela 2. Valores médios da resistência à compressão axial e para o módulo de elasticidade (MOE) e módulo de ruptura (MOR) à flexão estática da madeira de Toona ciliata aos 6, 12 e 18 anos de idade.

Table 2. Average values of compressive strength and modulus of elasticity (MOE) and modulus of rupture (MOR) in static bending of Toona ciliata wood at 6,12 and 18 years old.

\begin{tabular}{lccc}
\hline \multirow{2}{*}{ Idade (anos) } & \multicolumn{3}{c}{ Propriedades mecânicas } \\
\cline { 2 - 4 } & \multirow{2}{*}{ Compressão axial (MPa) } & MOR (MPa) & MOE (MPa) \\
\cline { 3 - 4 } & $24 \mathrm{~b}^{*}$ & $40 \mathrm{c}$ & $4634 \mathrm{~b}$ \\
6 & $(2,04)(8,38)^{* *}$ & $(4,65)(11,71)$ & $(456,29)(9,85)$ \\
\multirow{2}{*}{12} & $27 \mathrm{a}$ & $47 \mathrm{~b}$ & $5240 \mathrm{a}$ \\
& $(3,72)(13,59)$ & $(4,86)(10,40)$ & $(537,75)(10,26)$ \\
18 & $29 \mathrm{a}$ & $51 \mathrm{a}$ & $5441 \mathrm{a}$ \\
& $(4,55)(15,58)$ & $(4,81)(9,35)$ & $(485,02)(8,91)$ \\
\hline \multirow{2}{*}{ Média Geral } & 27 & 46 & 5105 \\
& $(4,10)(15,23)$ & $(6,77)(14,73)$ & $(598,83)(11,73)$ \\
\hline
\end{tabular}

*: As médias seguidas pela mesma letra não diferem estatisticamente entre si (Tukey, p >0,05). **: Valores entre parênteses são desvio padrão (Mpa) e coeficiente de variação (\%), respectivamente.

Os dados apresentados na tabela 2 evidenciaram um aumento nos valores médios da resistência à compressão axial, do MOE e MOR à flexão estática em função da idade. Pode-se observar, para a resistência à compressão axial, um valor médio de $27 \mathrm{MPa}$, em que estatisticamente as árvores aos 12 e 18 anos apresentaram os maiores valores, similares aos encontrados por Nassur (2010), que foi de 27,36 MPa.

Os valores médios encontrados para o MOR e MOE à flexão estática da madeira foram de $46 \mathrm{MPa}$ e $5105 \mathrm{MPa}$, respectivamente. Constatou-se que houve diferença significativa do módulo de ruptura entre as diferentes idades. Já para o módulo de elasticidade, as árvores aos 12 e 18 anos apresentaram os maiores valores, estatisticamente iguais. Nassur (2010) encontrou para o MOR um valor inferior de 29,02 MPa e superior para o MOE de $8198 \mathrm{MPa}$, quando comparados aos valores encontrados no presente estudo.

Ao se comparar a resistência à compressão axial e o módulo de elasticidade e ruptura à flexão estática da madeira com algumas espécies citadas na literatura (MAINIERI; CHIMELO, 1989; MORESCHI, 2005), constata-se que a madeira de Toona ciliata apresenta baixa resistência mecânica, o que pode ser justificado também pela diferença da idade do material.

A partir do beneficiamento da madeira de cedro australiano, podem-se obter diversos produtos e subprodutos destinados a diferentes fins. Contudo, os valores médios para a resistência mecânica encontrados são considerados baixos, o que implica a limitação do uso da madeira, principalmente em situações que exijam maiores esforços mecânicos, como é o caso da utilização para fins estruturais.

No que diz respeito ao cedro nativo do Brasil, do gênero Cedrela, deve-se ter cuidado ao utilizar o cedro australiano em sua substituição, uma vez que este último apresenta propriedades físicas e mecânicas inferiores. É importante levar em consideração que, em geral, madeiras provenientes de plantios florestais apresentam idades inferiores às árvores de floresta nativa, o que influencia diretamente nas propriedades físicas e mecânicas da madeira.

\section{CONCLUSÕES}

Com base nos resultados apresentados para as características da madeira de cedro australiano estudadas, podem-se tirar as seguintes conclusões:

- A densidade básica média foi classificada como madeira leve e crescente em função da idade.

- Com exceção da contração radial, as demais contrações reduziram em função do aumento da idade.

- A madeira apresentou uma estabilidade dimensional intermediária em função do seu coeficiente de retratibilidade volumétrica.

- O fator anisótropico tende a diminuir com o aumento da idade, o que pode proporcionar à madeira adulta melhor estabilidade dimensional.

- Em situações que exijam elevada resistência mecânica, o uso da madeira deve ser limitado.

- De maneira geral, a madeira de Toona ciliata obteve melhores valores para as propriedades físicas e mecânicas avaliadas com o aumento da idade, sendo a idade uma variável de suma importância na utilização final da madeira. 


\section{AGRADECIMENTOS}

Ao CNPq, pela concessão de bolsa de produtividade em pesquisa a um dos autores.

\section{REFERÊNCIAS}

ALMEIDA, A. A. Biodegradação de produtos à base da madeira de cedro australiano (Toona ciliata M. Roem. var. australis). 102 f. Dissertação (Mestrado em Ciências e Tecnologia da Madeira) Universidade Federal de Lavras. Lavras, 2010.

ARES, A.; FOWNES, J. H. Productivity, nutrient, and water-use efficiency of Eucalyptus saligna and Toona ciliata in Hawaii. Forest Ecology and Management. Amsterdam,v. 139, p. 227 - 236, 2000.

ASSOCIAÇÃO BRASILEIRA DE NORMAS TÉCNICAS (ABNT). Ensaios físicos e mecânicos de madeiras - método brasileiro. MB-26/1940. Rio de Janeiro: 1940. 16 p.

. NBR 7190: projeto de estruturas de madeiras. São Paulo, 1997.

BURGER, L. M.; RICHTER, H. G. Anatomia da madeira. São Paulo: Nobel; 1991.154 p.

CALEGARI, L.; GATTO, D. A.; SANTINI, E. J. Efeitos da temperatura de secagem sobre algumas propriedades em madeira juvenil e adulta de Pinus elliottii Engelm. In: CONGRESSO BRASILEIRO DE INDUSTRIALIZAÇÃO DA MADEIRA E PRODUTOS DE BASE FLORESTAL, 1, 2004, Pinhais, PR. Anais... Pinhais, PR: Docpar, 2004.

CUNNINGHAM, S. A.; FLOYD, R. B.; GRIFFITHS, M. W.; WYLIE, F. R. Patterns of host use by the shoot-borer Hypsipyla robusta (Pyralidae: Lepidoptera) comparing five Meliaceae tree species in Asia and Australia. Forest Ecology and Management. Amsterdam, v. 205, p. 351 - 357, 2005.

DURLO, M. A.; MARCHIORI, J. N. C. Tecnologia da madeira: retratibilidade. Santa Maria: CEPEF/FATEC, 33 p, 1992 (Série Técnica, 10).

FOREST PRODUCTS LABORATORY - Standard terms for describing wood. USDA. Forest Service. Forest Products Laboratory Research Paper. Madison, v. 0171, p. 1 - 10, 1973.

GONZAGA, A. L. Madeira: uso e conservação. Brasília, Distrito Federal. 2006. Disponível em: < http:// www.monumenta.gov.br/upload/Caderno\%20Madeiraweb_1173383037.pdf >. Acesso em: 04/01/2013.

GONÇALEZ, J. C.; BREDA, L. C. S.; BARROS, J. F. M.; MACEDO, D. G.; JANIN, G.; COSTA, A. F.; VALE, A. T. Características tecnológicas das madeiras de Eucalyptus grandis W. Hill ex Maiden e Eucalyptus cloeziana $\mathrm{F}$. Muell visando ao seu aproveitamento na indústria moveleira. Ciência Florestal, Santa Maria, v. 16, p. 329 - 341, 2006.

GONÇALVES, F. G.; OLIVEIRA, J. T. S. Resistência ao ataque de cupim-de-madeira-seca (Cryptotermes brevis) em seis espécies florestais. Cerne, Lavras, v. 12, p. 80 - 83, 2006.

HELLMEISTER, J. C. Sobre a determinação das características físicas da madeira. 119 f. Tese (Doutorado em Engenharia) - Universidade de São Paulo. São Carlos, 1982.

HUMPHREYS, R. D.; CHIMELO, J. P. Comparação entre propriedades físicas, mecânicas e estereológicas para agrupamento de madeiras. In: CONGRESSO NACIONAL SOBRE ESSÊNCIAS NATIVAS, 1992, São Paulo. Anais... São Paulo, 1992. p. 480 - 490.

KOLLMANN, F. F. P.; CÔTÉ JUNIOR, W. A. Principles of Wood Science and Technology. Berlin: Springer-Verlag, 1968. $592 \mathrm{p}$.

LAMPRECHT, H. Silvicultura nos trópicos. Eschoborn: GTZ, 1990. 343 p.

MAINIERI, C.; CHIMELO, J. P. Fichas de características das madeiras brasileiras. São Paulo: Instituto de Pesquisas Tecnológicas. 1989. 418 p.

MENDES, C. J. Influência da idade em Pinus taeda L. sobre a qualidade de madeira para a produção de celulose. Série Técnica IPEF, Piracicaba, v. 9, p. 81 - 90, 1993.

MORESCHI, J. C. Propriedades tecnológicas da madeira. Curitiba, 2005.169 p. (Manual didático). 
MORI, C. L. S.; MORI, F. A.; MENDES, L. M.; SILVA, J. R. M. Caracterização da madeira de angicovermelho (Anadenanthera peregrina (Benth.) Spreng) para confecção de móveis. Brasil Florestal, Brasília, p. 29 - 36, 2003.

NASSUR, O. A. C. Variabilidade das propriedades tecnológicas da madeira de Toona ciliata $\mathbf{M}$. Roem. com dezoito anos de idade. 84 f. Dissertação (Mestrado em Ciência e Tecnologia da Madeira) Universidade Federal de Lavras. Lavras, 2010.

OLIVEIRA, J. T. S. Caracterização da madeira de eucalipto para a construção civil. $429 \mathrm{f}$. Tese (Doutorado em Engenharia) - Universidade de São Paulo. São Paulo, 1997.

OLIVEIRA, J. T. S.; TOMAZELlO FILHO, M.; FIEDLER, N. C. Avaliação da retratibilidade da madeira de sete espécies de Eucalyptus. Revista Árvore, Viçosa, v. 34, n. 5, p. 929 - 936, 2010.

PANSHIN, A. J.; ZEEUW, C. de. Textbook of wood technology: structure, identification, uses, and properties of the commercial wood of the United States and Canada. New York: McGraw-Hill Book, 1980. v. 4.722 p.

PEREYRA, O.; SUIREZS, T. M.; PITSCH, C.; BAÉZ, R. Estúdio de las propriedades físico-mecánicas y comportamiento em procesos industriales de la madera de Kiri, Gravillea, Paraíso y Toona. Floresta, Curitiba, v. 36, p. 213 - 223, 2006.

PINHEIRO, A. L.; RAMALHO, R. S.; BARREIROS, H. S. Árvores exóticas em Viçosa: II. Toona ciliata M. Roem. var. australis (F. V. M.) C. DC. (MELIACEAE). Ceres, Viçosa, v. 41, p. 103 - 112, 1994.

RAYMOND, C. A. Tree breeding issues for solid wood products. In: Conference on The Future of Eucalypts for Wood Products. Proceedings of an IUFRO Conference', Launceston, Tasmania, IUFRO, 2000, p. 265 - 270.

SÁ, V. A. Potencial da madeira de cedro australiano (Toona ciliata M. Roem. var. australis) na manufatura de produtos de maior valor agregado. $103 \mathrm{f}$. Dissertação (Mestrado em Ciência e Tecnologia da Madeira) - Universidade Federal de Lavras. Lavras, 2009.

SCANAVACA JÚNIOR, L.; GARCIA, J. N. Determinação das propriedades físicas e mecânicas da madeira de Eucalyptus urophylla. Scientia Forestalis, Piracicaba, v. 65, p. 120 - 129, 2004.

SHIMOYAMA, V. R. S.; BARRICHELO, L. E. G. Influência de características anatômicas e químicas sobre a densidade básica da madeira de Eucalyptus spp. In: CONGRESSO ANUAL DE CELULOSE E PAPEL, 1991, São Paulo .Anais... São Paulo: ABTCP: 1991. p. 23 - 36.

SHIMOYAMA, V. R. Variações da densidade básica e características anatômicas e químicas da madeira em Eucalyptus sp. 93 f. Dissertação (Mestrado em Ciência Florestal) - Escola Superior de Agricultura Luiz de Queiroz. Piracicaba, 1990.

SORAGI, L. C. Qualidade de superfícies usinadas em madeira de Toona ciliata M. Roem. 71 f. Dissertação (Mestrado em Ciência e Tecnologia da Madeira) - Universidade Federal de Lavras. Lavras, 2009.

STANGERLIN, D. M; MELO, R. R.; DOMINGUES, J. M. X. Determinação da resistência ao impacto para as madeiras de Eucalyptus dunnii, Corymbia citriodora e Pouteria pachycarpa. In: Encontro Brasileiro em Madeira e Estruturas de Madeira. Anais... [CD-ROM], 2008, Londrina.

STURION, J. A.; PEREIRA, J. C. D.; ALBINO, J. C.; MORITA, M. Variação da densidade básica da madeira de doze espécies de Eucalyptus plantadas em Uberaba, MG. Boletim de Pesquisa Florestal, Colombo, n. 14, p. 28 - 38, 1987.

TRIANOSKI, R. Avaliação do potencial de espécies florestais alternativas de rápido crescimento, para produção de painéis de madeira aglomerada. $260 \mathrm{f}$. Dissertação (Mestrado em Engenharia Florestal) - Universidade Federal do Paraná. Curitiba, 2010.

ZIECH, R. Q. S. Estudo das características tecnológicas da madeira de cedro australiano (Toona ciliata M. Roem.). 74 f. Dissertação (Mestrado em Ciência e Tecnologia da Madeira) - Universidade Federal de Lavras. Lavras, 2008. 